\title{
Co-production and Co-creation: Creative Practice in Social Inclusion
}

\author{
Atau Tanaka ${ }^{1}$, Lalya Gaye ${ }^{1}$, and Ranald Richardson ${ }^{2}$ \\ ${ }^{1}$ Culture Lab \\ ${ }^{2}$ Centre for Urban Regional Development Studies \\ Newcastle University \\ NE1 7RU Newcastle upon Tyne, United Kingdom \\ \{atau.tanaka, lalya.gaye, ranald.richardson\}@ncl.ac.uk
}

\begin{abstract}
We apply techniques drawn from interactive media art in fieldwork for social inclusion. Advanced mobile media and grassroots DIY techniques are used to bring creative practice with digital media into community based outreach work. We use these techniques in a participatory context that encourages the co-production of cultural output. We triangulate across artistic practice, technology engineering, and the social sciences to leverage methods from digital media art practice in contexts that result in social innovation.
\end{abstract}

Keywords: interactive music, social inclusion, social innovation, co-production of knowledge, triangulation.

\section{Introduction}

Interactive media arts practice has developed in the last quarter century from a highly specialized field that required high-end machines, to an increasingly democratized field of practice carried out on everyday technology. This history and progression of technology, aesthetic, and practice, can be retraced in the history of the festival Ars Electronica, and is chronicled in books such as $[1,2]$. Media art can be characterized as creative artistic practice that makes use of digital technologies in mixed media settings including visual and sound media in interactive situations that invite varying degrees of spectator interaction.

Artists have responded to developments in technology in critical and embracing ways. Two important developments in the last twenty years include the rapid miniaturization and accompanying democratization of technology, and the arrival of wide public use of the Internet. The former is seen in the increasing power of laptop computers and mobile devices to perform numerical calculations and signal processing for graphics or audio rendering - tasks that earlier in the history of the field required mainframe computers. The availability of this calculation power on portable computers changes the cultural contexts in which media art is practiced - it can leave the studio and large arts center and be situated in public space and in the wild. Increased availability also brings with it lowered barriers of access, broadening both the range 
of artists practicing media art, and the ways in which works of media art are able to invite audience participation.

In parallel with the democratization of technology came the widespread take up of Internet technologies in all aspects of daily life. This has resulted in artists using the Internet as a new form of communication and distribution for their work. It has also spawned new art forms and artistic movements, notably that of net.art [3], artwork conceived for and delivered to a standard web browser.

We can characterize media art of having 4 distinct characteristics

1. Interaction

2. Sensor input

3. Malleable Media

4. DIY ethos

In the work described here, we draw upon techniques and methods from one specific area of media art practice, that of interactive music. Computer music is the field that emerged from the invention of digital audio in the 1950's [4, 5]. It elaborates techniques of sound synthesis, computer-aided musical composition, and representation that today are at the heart of the CD audio standard, generative game soundtracks, and perceptual coding techniques like MP3. One branch of computer music that is directly concerned with human-computer interaction is that of New Interfaces for Musical Expression (NIME). NIME began as a workshop at CHI 2001 [6] and has since evolved to sustain an annual international conference. As its nature implies, NIME is the discipline concerned with interaction in music. It is a field specialized in creating interactive music systems that use sensors and networks. Its activities can be described as those of instrument building, or lutherie. The typical context of a NIME system is to build a system for concert performance. A NIME system is conceived by an instrument builder, contextualized by a composer, to be used by a musician who is able to attain a profound level of interaction with the system through his/her virtuosity. As a field of practice, NIME has been specialist oriented. Recently, however, the increasing use of sensing technologies in consumer electronics, typified by the Nintendo Wii-mote controller with its accelerometers, have meant that interactive music practice has the potential to become more widespread.

\section{Social Inclusion}

Inclusion, in the social sciences, is most often described to counter the effects of social exclusion. Social exclusion is a complex process of alienation from mainstream society, detaching groups and individuals from relations and institutions and preventing full participation in the normal advancement of the society in which they live [7].

Poor health, disability, family breakdown, poverty and unemployment are just some of the reasons why people of all ages may become marginalized from society. These multiple symptoms prevent individuals or groups in benefitting from the economic, social, and political life around them. These effects are often self-reinforcing and self-perpetuating. 


\subsection{Digital Inclusion}

Digital technologies represent, at once a possible solution to social exclusion, and a feature of mainstream society that makes inclusion all the more difficult to attain. The digital divide is the separation between those in society with access to information technologies and those without $[8,9]$. Transforming information and communications technologies (ICT) from an exclusionary aspect to a society to an inclusionary force typically focus on questions of lowering barriers to access $[10,11]$.

Currently, the potential benefits of the information society are not being realized by all members of society; it is recognized that digital exclusion mirrors many aspects of the more general social exclusion described above. That is, not just poverty, but the mutually reinforcing consequences of citizens enduring unemployment, discrimination, poor housing, crime, bad heath and family breakdown.

Social innovation is broadly defined as forms strategies and actions that have direct benefit in civil society in education, community development, workplace conditions, and health [12]. Here it is relevant as a way to conceptualize the potential benefit of technology and creative practice on the socioeconomic empowerment of young people.

\subsection{Social Inclusion through the Digital Economy}

The 'Social Inclusion through the Digital Economy' (SiDE) research hub [13] is a project funded by the UK Research Council Digital Economy Programme that applies developments in ICT to social benefit. It aims to actively explore the transformative potentials of new technologies for individuals and communities at risk of or suffering from social exclusion. For that purpose, it addresses four fields where digital technologies may deliver major social benefits:

1. Assistive technologies in domestic environments

2. Accessibility of ICT to broader age groups

3. Inclusive Transport Services

4. The Creative Industries

SiDE's contention is that it is necessary to work with end-users in a sustained way, to understand their situation, environment and needs. Our multidisciplinary teams of researchers have access to large user groups affected by social exclusion, including a group of 3000 volunteers, containing people from a range of age groups and with a variety of social backgrounds and forms of exclusion. SiDE is also centered on the formation of communities of practice of social inclusion stakeholders, which include academics, practitioners, technology producers and those who are, or who feel themselves to be, excluded from parts of society. In establishing such a community, SiDE seeks not just to create products and applications to assist users in ameliorating social disadvantage, but to establish inclusionary processes to help excluded people participate in society. In this process, SiDE seeks to broaden the horizons, capacities and understanding of all partners and contribute to formulating future policy on a socially inclusive digital society. 


\section{Creative practice and Co-creation}

The Creative Media Group represents one of the four strands of research within the SiDE project, the Creative Industries. It works specifically with creative arts practices and young people in methodologies of co-creation to combat effects of marginalization. This paper describes the work of the Creative Media group deploying digital technologies in fieldwork with marginalized youth, in collaboration with regional youth work organizations.

Creative practice encapsulates process and methods from the creative arts, in our case using interactive media technologies. The research hypothesis here is:

- Creative media arts practice represents forms of deep interaction with digital technologies not encountered in typical end-user usage of ICT

- That this deeper view of digital media as accessible and malleable create opportunities for democratizing the creative process itself

- In order to realize this vision, that arts skills can be transferred to a general public for broader benefit and impact

Creative skills serve three purposes in the context of this project: 1) as skills that make young people entering the workforce more competitive, 2) as critical cerebral activity to develop and maintain mental acuity, 3) as compelling cultural activity to engage disaffected youth.

Co-production is a broad term encompassing fields of activity ranging from cultural production to knowledge and skills, to business applications. Here we introduce the generalities of how the term is used in these respective fields, and from that establish a transdisciplinary, synthetic view that we apply as a means to bridge creative media art practice and community based work in social inclusion.

Co-production in the cultural sector typically refers to the collaboration of two organizational entities in the realization of a new artwork. It thus refers to the consolidation of means as a way to facilitate the production of ambitious, large-scale work that could not be supported by one venue.

The use of terms co-creation and co-production in the field of human computer interaction research typically refer to the inclusion of non-designers or non-engineers in an interaction design or technology development cycle [14]. Methods that implement dynamics of co-production in interaction design include User Centered Design (UCD), and Participatory Design (PD) [15]. These methods include the end user in the design process through techniques of structured brainstorming and ethnographically inspired forms of qualitative data collection, with the goal to better understand the actual people who will use the systems being designed.

Co-production of knowledge is used in both a social science as well as business development context to refer to ways, be they exploitative or altruistic, where two parties in a hierarchical relationship, often of establishment and individual, arrive at forms of mutual understanding [16]. In scientific knowledge production, coproduction of knowledge was codified as Mode 2 as a form of transdiscplinary, level field form of information sharing for scientific advancement [17]. We draw upon the social science literature where inclusive knowledge production contexts are used to facilitate social innovation. Co-production facilitated by digital technologies has been covered in $[18,19]$. 
We were interested to apply the end user orientation of user centered design from $\mathrm{HCI}$ and the democratic ideals of coproduction of knowledge for social innovation to conceive of new processes of creative practice. This is most closely associated with developments in the Do It Yourself (DIY) movement. We call this participatory art practice and draw upon political texts of the 1960's to inform this [20,21].

\section{Triangulation across Disciplines}

Triangulation is one approach to cross disciplinary research. It fulfills three interdisciplinary goals: the social science goal of understanding the needs and desires of users in a real-world setting, the engineering goal of field- testing the technology, and the design goal of inspiring users and researchers to think about new technologies [22]. By forming a perspective from related disciplines, it has the potential to provide a deeper understanding of a design problem. We establish a process of exchange between arts practice, technology engineering, and social science to build advanced creative technologies while confirming everyday usage of existing technologies and by doing so put in place parallel streams that intertwine and balance usability with musicality. We call upon the notion of triangulation to leverage the complementarities of music research and user centered design to create inclusive creative situations that can be studied through the lens of social science.

\section{Methods: A Music Scenario}

We engage with cultural sector partners with established outreach activities to identify and access groups of young people from difficult neighbourhoods, where school success rates or employment are low. We planned workshops where interactive technology is deployed in acts of creative practice. The general workshop plan was conceived to have several phases:

1. Acclimatisation - getting to know the group, asking them what kinds of music they like, having them come up to the front and showing the group websites of their favorite music

2. Basic Sampling - introducting audio editing software to show how music can be used not just to copy and share, but as the source material for basic content editing: cutting, pasting, looping

3. Advanced Tools - we then introduce advanced computer music composition tools we will develop in the project. This software democratizes heretofore specialist production techniques so that will run on accessible hardware including mobile phones

4. User Sampler Instrument - samples edited and saved during phase 2 will be loaded into the software introduced in phase 3 to allow the user to create their own sound sample playback instrument, being able to scratch, scrub, trigger, and pitch shift sounds through various interaction modalities such as the touchscreens and tilt-sensors of mobile phones

5. Publishing - once each user has created and saved a remix of music they listen to, it will be uploaded the SiDE website that allows exchange of media files 
6. Peer Commentary - we plan to use the peer critique dynamic to allow users to comment on each other's work. This sensitizes them to use creative practice as a medium through which to give constructive criticism, as a means to teach by example positive communication as opposed to anti-social slander.

7. Virtual Music Economy - Once a body of work is created by the user group, it becomes a catalogue of creative cultural output that represents that community. We could imagine a virtual economy of credits and tokens for "purchase" and "exchange" of the contents created by the users.

\section{Results}

Based on this research work plan, we conducted a pilot study with our first partner, Generator Music. Generator is a leading regional and national agency for the development of popular music. Among other things, Generator provides a variety of programs for regional music business development, industry skill-building, and for the support for emerging and aspiring young musicians. One of the programs covered by Generator is an Urban Music Training (UMT) program for aspiring young musicians (from DJs to instrumentalists and vocalists) which supports them in creating, recording and performing their own music, as well as helps them organize and promote events and provides them with professional level training for entering the music industry. Generator tends to target youth from difficult neighborhoods who would benefit the most from engaging in such a program. Young people enrolled in the program represent a wide diversity of ethnic and educational backgrounds and ages. Generator encourages musical innovation and supports high-quality professional level endresults that the young people can take pride in and potentially enter the music business. UMT thus displays an aim to nurture the young people's self-esteem and open up professional opportunities. One of the UMT classes called UMT: BEATS is targeted towards DJs and urban music producers and runs twice a week after school hours over a period of 12 weeks. Another one called UMT: PLAY is focused on instrument playing in band formations, and runs each year for a week, full-time. Each program ends with a public performance in settings of professional standard.

Our collaboration with Generator consisted of contributing to the UMT program by exposing young people to interactive music technologies, as well as designing participatory activities aimed towards technology-supported creative engagement in physical and community space. The first part of the process in working with UMT was an approach phase consisting of reciprocal visits, demos and focused discussions that have lead to potential workshop ideas. This was followed by a short planning phase that resulted in a proposal for a 'Remix Your Instrument' pilot workshop, which we delivered shortly thereafter. The workshop took place during UMT: PLAY and aimed to build on the young people's existing musical skills. At the same time, it aimed to open a new world of possibilities and musical innovation to them and make a connection between urban electronic music and the physicality of instrument playing. Split into groups, the young people got to experiment with augmenting music instruments with sensors (pressure, light intensity, bend, movement etc) that modified the sounds that they produced. We used open-source, easily programmable hardware and software that we packaged in simple and approachable modules, in order to let the young 
people quickly learn how to use them while giving them a have chance to modify them themselves. Young people in each group collaborated to make music: playing instruments, using the sensor they were provided with, and modifying sound effects. Some even used their own mobile phones, voices and other resources at hand in the process. This workshop was successful in getting the young people excited and engaged in playing with innovative interactive music technology. One group was even eager to continue playing with the technology after the workshop and kept its module for the rest of week.

The next activities will take place over a longer period of time, within the UMT: BEATS training program. They will consist of three workshops centered on the use of mobile phones and MP3 players; technologies that young people are fairly familiar with and enjoy using [23]. These 'Remix Your 'Hood' (neighborhood) workshops will make use of RJDJ [24], an off-the-shelf free reactive music application that remixes ambient sounds into music in real time, in a way related to e.g. [25], typically through headphones. This enables one to see their own environment with new eyes, engage with it, and be creative with it in context. Various so-called 'scenes' (sound processing options) are available in RJDJ, together with the possibility to record the resulting music and share it online. Here as well, there is a DIY dimension to the technology: it is built on top of an open-source environment, which allows one to create their own scenes. Workshops are currently sketched out to be the following: 1) sound-walks with existing RJDJ scenes and brainstorming design session; 2) a programming session for composing one's own scenes; 3) a "make your own speakers/sound-parasites" DIY hacking workshop for turning any surface into a speaker - from junk boxes to windows in urban space. The last workshop will end with a performance in public space, with the everyday environment used as a resource for creation and an interface for performance. Besides taking pride in the creative potentials of the mundane of their neighborhood environment, this process aims to foster locally-rooted and original musical innovation which may give a sense of "representing" one's own community.

Throughout our collaboration with Generator in this phase of the SiDE project, we are interested in observing how young people interact with such creative interactive technologies and appropriate them, as well as explore how this makes them engaged in their environment and with others. Based on findings from this, the following phase will transition to the participatory design of interactive prototypes for creativity and social inclusion of young people, a process where the input of the participants will help them bring social capital in technology development that will be deployed at the scale of the whole region.

\section{Discussion}

It was important to create a strategy of partnership that could connect our work in SiDE with ongoing regional inclusion work, in a way that would yield mutual benefit. Doing so brings several pragmatic benefits besides the deeper benefits of forging meaningful collaborative partnerships. These include ready access to established user groups, consultation with experienced and approved facilitators. 
Working with partners reveals a two-tiered structure, both institutionally and personally. Institutionally, the objects of study become as much the partner institution as the user group assembled by the partner. The latter in this view can be seen as "institutional" as it is an entity whose makeup is defined by the organizations in question and in this way are distinct from any group of users SiDE would assemble directly or that might emerge spontaneously.

The effectiveness of a working relationship is often influenced by institutional agendas. Community outreach and cultural sector organizations do not work in a research context, so the timescales for work and depth of enquiry are limited in this regard. Creating a fruitful research partnership where research extracts from the activity meaningful results is a not insignificant challenge.

At the user level, a two-tiered differentiation of beneficiaries emerges. Mediators and facilitators of the existing outreach activities have emerged as a crucial component in the delivery structure. The end user is the young person taking part in the workshop activity.

This has brought about an interesting dynamic in the participatory design activities. Facilitators are often quick to grasp the potential of SiDE technology and imagine the enhancement of activities they deliver. End users may lack the confidence to express themselves or think through a hunch or question they may have, but that creating an environment conducive to non-hierarchical discussion can bring out unexpected results.

The wish to compartmentalize these levels of user to better understand them is a natural tendency that brings up significant problems. It is attractive as a means to protect the end user from any institutional agenda that is represented by the presence of the mediator. However, ease of access, and the dynamics of trust would necessarily be impacted by isolating these user types.

Strategies for establishing partnerships and carrying out collaborative work feed directly into the research methodology. This can be summarized as:

1. Establishing criteria for partnership selection based on existing work, social benefit, and the potential for enhancement through digital technology

2. Identifying contribution to be made by SiDE through observation, participatory discussion, and pilot studies

3. Develop contribution by design, engineering, development, and deployment

4. Measure effect of contribution through observation, discussion, and analysis

5. Iterate

\section{Conclusion}

We have described the first field trial of the Creative Media group in the large scale project, Social Inclusion through the Digital Economy. In this trial, we partnered with Generator Music, a regional music development charity with an existing user base of young people from Newcastle upon Tyne. We put in place a process of acclimatization and participatory consultation that led to the conception of music technology sessions that introduced interactive music techniques to enhance an existing workshop program. 
Our approach meant that the participants are working directly with materials that are the music they listen to. They re-appropriate and become pro-active with media they heretofore only passively consumed. The user group here, young people, represent an interesting challenge in digital inclusion and accessibility work. They are the born digital generation, so use of digital technology is not the problem. The problem is not technology use, but a sense of empowerment. Rather seeing mobile music as a consumer entertainment medium, we sought to communicate the simple message that with the right tools, music can be made interactive, and becomes a democratized medium for personal expression.

The enthusiasm that the young people demonstrated in the workshop sessions fulfills the hypothesis that novel forms of interaction with digital media can unlock access to creative practice processes for social benefit. This points to the possibility that from this we could identify forms of knowledge that are being shared that could result in the acquisition of transferrable skills that would permit the young people in question to engage more fully with broader aspects of contemporary digital society. In this way we consider that our approach has potential benefits in the area of social innovation.

This work applies the technique of triangulation across disciplines. We used participatory design methodology from human-computer interaction (HCI) research to introduce interactive music technologies from New Interfaces for Musical Expression (NIME) practice, and studied the outcomes from a social science perspective. This represented a form of action-based research situated in real life situations, in the wild. The use of advanced digital technologies was found to be attractive to both the young people as well as to the workshop facilitators. Carrying out the field trial hi-lighted higher level institutional differences between interactive media research and community arts outreach work that point to both potential benefits as well as issues to be resolved in future work. This provides initial results and reflection on the application of creative digital media practice in a social inclusion setting.

\section{References}

1. Schwarz, H.-P.: Media-Art-History: Media Museum. Prestel, Munich (1997)

2. Grau, O.: MediaArtHistories. MIT Press, Cambridge (2007)

3. Reisinger, G., Daniels, D.: Net Pioneers 1.0: Contextualizing Early Net-Based Art. Sternberg Press, Berlin (2010)

4. Dodge, C., Jerse, T.A.: Computer Music: Synthesis, Composition, and Performance. Schirmer Books, New York (1985)

5. Risset, J.-C.: Introductory Catalogue of Computer Synthesized Sounds. Bell Telephone Laboratories, Murray Hill (1969)

6. Poupyrev, I., Lyons, M.J., Fels, S., Blaine, T.: New Interfaces For Musical Expression Workshop. In: CHI 2001 Extended Abstracts (2001)

7. Silver, H.: Policies to combat social exclusion: A French-British comparison. International Institute for Labour Studies, Geneva (1995)

8. Compaine, B.M.: The Digital Divide: Facing a Crisis or Creating a Myth? MIT Press, Cambridge (2001)

9. Warschauer, M.: Technology and Social Inclusion: Rethinking the Digital Divide. MIT Press, Cambridge (2004) 
10. Katz, J., Rice, R.: Social Consequences of Internet Use: Access, Involvement, and Interaction. MIT Press, Cambridge (2002)

11. Digital Britain. Department for Culture, Media and Sport, Department for Business, Innovation and Skills, London (2009)

12. Dench, G., Flower, T., Gavron, K.: Young at Eighty. Carcanet, Manchester (1995)

13. Social Inclusion through the Digital Economy, http: / / www. side.ac.uk

14. Abras, C., Maloney-Krichmar, D., Preece, J.: User-Centered Design. In: Bainbridge, W. (ed.) Encyclopedia of Human-Computer Interaction. Sage Publications, Thousand Oaks (2004)

15. Vredenburg, K., Mao, J.Y., Smith, P.W., Carey, T.: A survey of user-centered design practice. In: Proceedings of the SIGCHI Conference on Human Factors in Computing Systems, pp. 471-478. ACM Press, New York (2002)

16. Jasanoff, S.: States of Knowledge: The Co-Production of Science and the Social Order. Routledge, Oxford (2006)

17. Gibbons, M., Limoges, C., et al.: The New Production of Knowledge: The dynamics of science and research in contemporary societies. Sage Publications, Thousand Oaks (1994)

18. Oudshoorn, N., Pinch, T.: How Users Matter: The Co-Construction of Users And Technology. MIT Press, Cambridge (2003)

19. Leadbeater, C.: We-think: Mass innovation, not mass production: The Power of Mass Creativity. Profile Books, London (2008)

20. Jo, K., Tanaka, A.: The Music Participates. In: Schroeder, F. (ed.) Performing Technology: User Content and the New Digital Media. Cambridge Scholars Publishing, Newcastle upon Tyne (2009)

21. Arnstein, S.R.: A Ladder of Citizen Participation. Journal of the American Institute of Planners 35(4), 216-224 (1969)

22. Mackay, W.E., Fayard, A.-L.: HCI, Natural Science and Design: A Framework for Triangulation Across Disciplines. In: Proc. DIS 1997, Amsterdam, The Netherlands (1997)

23. Unterfrauner, E., Marschalek, I.: ICT and Mobile Phones as Resources for Marginalised Youth. In: Proc. Interaction Design and Children, IDC 2009 (2009)

24. Reality Jockey, http://www.rjdj.me

25. Gaye, L., Mazé, R., Holmquist, L.E.: Sonic City: The Urban Environment as a Musical Interface. In: Proc. NIME (2003) 